\title{
Staphylococcus aureus Colonization and Long-Term Risk for Death, United States
}

\section{Angelico Mendy, Edgar R. Vieira, Ahmed N. Albatineh, Janvier Gasana}

To examine the association of colonization by Staphylococcus aureus and general population mortality, we followed 10,598 adults for 8.5 years on average. Methicillin-susceptible $S$. aureus colonization was not associated with death. Methicillin-resistant $S$. aureus carriage predicted death in a crude analysis but not after adjustment for socioeconomic status and co-morbidities.

Staphylococcus aureus is a common cause of mild to life$\checkmark$ threatening infections. It is differentiated into methicillin-susceptible $S$. aureus (MSSA) and methicillin-resistant $S$. aureus (MRSA); the emergence of isolates resistant to vancomycin has raised concern that the bacterium might become untreatable with current antimicrobial drugs (1). Whether $S$. aureus colonization influences general population mortality remains unclear $(2,3)$. Therefore, we examined the association in a US population representative sample followed for nearly a decade.

\section{The Study}

We used data from the 2001-2004 National Health and Nutrition Examination Survey (NHANES) and the NHANES 2001-2004 Mortality-Linked File. NHANES is a survey of the US noninstitutionalized civilian population conducted by the Centers for Disease Control and Prevention's National Center for Health Statistics. We selected 10,598 NHANES participants using a complex multistage sampling design and followed them through December 31, 2011, to track mortality. NHANES protocols were approved by the institutional review boards of NCHS and CDC, and all participants provided informed consent.

Nasal swabs collected from participants were tested for S. aureus and methicillin resistance during 2001-2004 following the National Committee for Clinical Laboratory Standards disk-diffusion method. We additionally tested isolates resistant to oxacillin (MRSA) and intermediately resistant to oxacillin and every tenth isolate sensitive to

Author affiliations: University of lowa, lowa City, lowa, USA

(A. Mendy); Florida International University, Miami, Florida, USA

(E.R. Vieira, J. Gasana); Kuwait University, Kuwait City, Kuwait (A.N. Albatineh)

DOI: http://dx.doi.org/10.3201/eid2211.160220 oxacillin (MSSA) for staphylococcal cassette chromosome mec (SCCmec) typing, genes encoding enterotoxins, toxic shock syndrome toxin-1, and Panton-Valentine leukocidin (PVL) toxin (details on the methods are available at http://www.cdc.gov/nchs/data/nhanes/nhanes_01_02/ 135_b_doc.pdf). We collected data on age, sex, race/ethnicity, family income, smoking, and medical conditions using questionnaires.

Univariate and multivariate Cox proportional hazards regressions were used to estimate the hazard ratio (HR) with corresponding 95\% CIs for MSSA and MRSA association with mortality. Analyses were performed in STATA version 11 (StataCorp LP, College Station, TX, USA), taking into account the survey design and sampling weights, to produce nationally representative estimates. We considered $\mathrm{p}$ values $<0.05$ as statistically significant.

A total of $27.3 \%$ of participants were colonized with MSSA and $1.3 \%$ with MRSA (Table 1). During an average of 8.5 years of follow-up, the total death rate per 1,000 person-years was 10.3: 10.8 for noncolonized participants, 8.8 for those colonized with MSSA, and 25.6\%for those colonized with MRSA. MRSA-colonized participants tended to be older and non-Hispanic white, have low poverty income ratio, smoke cigarettes, have co-morbidities, or have been hospitalized and/or have had dialysis in the previous 12 months (Table 1). Approximately 58.6\% of MRSA isolates were of SCCmec type II, suggestive of hospital-associated MRSA, and $41.4 \%$ had MRSA isolates of SCCmec type IV, indicative of community-associated MRSA.

In Cox regression, MSSA-colonized participants had a significantly lower risk for death than did noncolonized participants in univariate analysis (hazard ratio [HR] $0.82,95 \%$ CI $0.68-0.98$ ) but not after adjustment for covariates. MRSA colonization was associated with higher risk for death than no $S$. aureus colonization in univariate analysis (HR 2.40, 95\% CI 1.56-3.69) and after adjustment for sociodemographic factors (HR 1.54, 95\% CI 1.06-2.34); MRSA colonization also carried higher risk for death than MSSA colonization (HR 2.90, 95\% CI 1.92-4.40). However, after additional adjustment for smoking, body mass index, and co-morbidities (asthma, diabetes, hypertension, cardiovascular diseases, stroke, liver disease, and cancer), these associations were no longer significant (Table 2).

Likewise, colonization with SCCmec type II MRSA more strongly predicted long-term mortality than did no $S$. aureus ((HR 3.38, 95\% CI 2.00-5.72) or MSSA 
Table 1. Characteristics of study participants by Staphylococcus aureus colonization status, National Health and Nutrition Examination Survey, United States, 2001-2004*

\begin{tabular}{|c|c|c|c|c|c|}
\hline Characteristic & No S. aureus & MSSA & MRSA & $\mathrm{p}$ value & All participants, $\mathrm{N}=10,598$ \\
\hline Prevalence & 71.4 & 27.3 & 1.3 & & 100 \\
\hline Median age, y (IQR) & $43(31-56)$ & $41(30-54)$ & $54(34-67)$ & & $43(31-55)$ \\
\hline Sex & & & & $<0.001$ & \\
\hline M & 46.1 & 54.0 & 36.4 & & 48.1 \\
\hline $\mathrm{F}$ & 53.9 & 46.0 & 63.6 & & 51.9 \\
\hline Race/ethnicity & & & & $<0.001$ & \\
\hline Non-Hispanic white & 70.3 & 74.4 & 80.8 & & 71.5 \\
\hline Non-Hispanic black & 12.1 & 8.4 & 11.7 & & 11.1 \\
\hline Hispanic & 12.4 & 12.8 & 4.5 & & 12.4 \\
\hline Other & 5.2 & 4.5 & 3.0 & & 5.0 \\
\hline Body mass index & & & & .28 & \\
\hline Normal or underweight & 34.6 & 32.6 & 33.8 & & 34.0 \\
\hline Overweight or obese & 62.4 & 65.0 & 63.6 & & 63.2 \\
\hline Missing & 3.0 & 2.4 & 2.5 & & 2.8 \\
\hline Poverty income ratio & & & & $<0.001$ & \\
\hline$<1.85$ & 30.4 & 29.4 & 42.7 & & 30.3 \\
\hline$\geq 1.85$ & 64.1 & 64.3 & 47.7 & & 63.9 \\
\hline $\bar{M}$ Missing & 5.5 & 6.3 & 0.6 & & 5.8 \\
\hline Smoking & 49.2 & 44.6 & 63.8 & $<0.001$ & 48.1 \\
\hline \multicolumn{6}{|l|}{ Co-morbidities } \\
\hline Asthma & 11.9 & 12.9 & 20.5 & .07 & 12.3 \\
\hline Diabetes & 8.1 & 9.4 & 13.5 & .13 & 8.6 \\
\hline Hypertension & 33.0 & 30.3 & 39.5 & .05 & 32.4 \\
\hline Cardiovascular diseases $\ddagger$ & 6.1 & 4.6 & 12.5 & .01 & 5.7 \\
\hline Stroke & 2.7 & 1.9 & 4.9 & .03 & 2.5 \\
\hline Liver disease & 3.4 & 3.1 & 2.6 & .76 & 3.3 \\
\hline Cancer & 8.9 & 7.3 & 16.7 & .02 & 8.5 \\
\hline Hospitalization and/or dialysis in past $12 \mathrm{mo}$ & 10.5 & 10.6 & 19.8 & $<0.001$ & 10.6 \\
\hline Mean survival, y (SE) & $8.47(0.11)$ & $8.67(0.10)$ & $7.5(0.28)$ & $<0.001$ & $8.52(0.10)$ \\
\hline Death during follow up & 9.1 & 7.7 & 19.3 & $<0.001$ & 8.9 \\
\hline \multicolumn{6}{|c|}{$\begin{array}{l}{ }^{*} \text { All values are percentages except as indicated. IQR, interquartile range; MSSA, methicillin-susceptible } S \text {. aureus; MRSA, methicillin resistant } S \text {. aureus. } \\
\dagger p \text { values for comparison of non-S. aureus colonization vs. MRSA colonization vs. MSSA colonization; } p \text { values calculated using } \chi^{2} \text { test for categorical } \\
\text { variables and analysis of variance for continuous variables. } \\
\ddagger \text { Coronary heart disease, congestive heart failure, and myocardial infarction. }\end{array}$} \\
\hline
\end{tabular}

colonization (HR 4.05, 95\% CI 2.42-6.79) in univariate analyses but not after adjustment for sociodemographic characteristics. Colonization with SCCmec type IV MRSA was not associated with death in univariate or adjusted analysis, unlike no $S$. aureus or MSSA colonization (Table 2). However, the small number of participants with MRSA SCCmec types II (83) and IV (53) limited our analysis.

\section{Conclusions}

In this population-based study, we found that MSSA is not associated with long-term mortality. MRSA is associated with risk for death in crude analysis but not after adjustment for socioeconomic factors, co-morbidities, or both.

Regarding MSSA, our results are consistent with studies conducted in inpatient and nursing home participants that also failed to find an association (4-7). Many of these studies were underpowered, calling for larger scale investigations. In line with the inverse association in our unadjusted analysis for MSSA and mortality, Wertheim et al. found that MSSA-colonized participants had longer survival than did noncolonized participants among patients with $S$. aureus bacteremia (3). They suggested that colonized patients might be more immunologically adapted to the endogenous $S$. aureus strains that caused the bacteremia or that exogenous strains in noncolonized patients were more virulent. In our case, we hypothesized that the negative association was imputable to some confounders, such as race/ethnicity, smoking, and co-morbidities (hypertension, cardiovascular diseases, stroke), instead of an independent association. We found that persons with MSSA were more likely to be non-Hispanic white and have asthma or diabetes than were persons without $S$. aureus nasal carriage, who were more likely be non-Hispanic black; have a lower poverty income ratio; be a smoker; and have hypertension, cardiovascular diseases, stroke, liver disease, or cancer. Concerning MRSA, Chan et al. observed that MRSA colonization was associated with higher mortality than non-MRSA colonization in nursing home residents in China followed for 2 years (8). Previous research also found that methicillin resistance in $S$. aureus bacteremia did not predict long-term mortality after adjustment for age, comorbidities, severity of acute illness, metastatic infections, and long-term facility resident status (1). 
Table 2. Cox proportional hazards regression for Staphylococcus aureus colonization and long-term mortality, United States*

\begin{tabular}{|c|c|c|c|c|c|c|c|c|}
\hline \multirow[b]{2}{*}{ Exposure variableł } & \multirow{2}{*}{$\begin{array}{c}\text { No. } \\
\text { deaths }\end{array}$} & \multirow{2}{*}{$\begin{array}{c}\text { No. } \\
\text { patients }\end{array}$} & \multicolumn{2}{|c|}{ Unadjusted } & \multicolumn{2}{|l|}{ Model 1} & \multicolumn{2}{|l|}{ Model 2} \\
\hline & & & $\mathrm{HR} \dagger(95 \% \mathrm{Cl})$ & $p$ value & $\mathrm{HR}+(95 \% \mathrm{Cl}) \S$ & $p$ value & HR† $(95 \%$ Cl) & $p$ value \\
\hline \multicolumn{9}{|l|}{ S. aureus colonization } \\
\hline No S. aureus & 1,051 & 7,794 & 1 (referent) & & 1 (referent) & & 1 (referent) & \\
\hline MSSA & 279 & 2,668 & $0.82(0.68-0.98)$ & 0.035 & $1.03(0.85-1.24)$ & 0.79 & $1.06(0.88-1.27)$ & 0.52 \\
\hline MRSA & 41 & 136 & $.40(1.56-3.69)$ & $<0.001$ & $1.54(1.06-2.34)$ & 0.026 & $1.38(0.88-2.17)$ & .15 \\
\hline MRSA SCCmec II & 36 & 83 & $.38(2.00-5.72)$ & $<0.001$ & $1.42(0.88-2.26)$ & 0.14 & $1.40(0.87-2.24)$ & 0.16 \\
\hline MRSA SCCmec IV & 5 & 53 & $1.07(0.37-3.12)$ & 0.9 & $2.05(0.60-7.03)$ & & $1.96(0.55-6.92)$ & 0.29 \\
\hline \multicolumn{9}{|l|}{ In colonized participants } \\
\hline MSSA & 279 & 2,668 & 1 (referent) & & 1 (referent) & & 1 (referent) & \\
\hline MRSA & 41 & 136 & $2.90(1.92-4.40)$ & $<0.001$ & $1.48(0.94-2.32)$ & 0.09 & $1.27(0.76-2.13)$ & 0.35 \\
\hline MRSA SCCmec II & 36 & 83 & $4.05(2.42-6.79)$ & $<0.001$ & $1.36(0.84-2.21)$ & 0.2 & $1.17(0.66-2.08)$ & 0.58 \\
\hline MRSA SCCmec IV & 5 & 53 & $1.30(0.44-3.84)$ & 0.63 & $1.90(0.54-6.64)$ & 0.3 & $1.97(0.58-6.70)$ & 0.26 \\
\hline \multicolumn{9}{|c|}{$\begin{array}{l}\text { *HR, hazard ratio; MSSA, methicillin-susceptible S. aureus; MRSA, methicillin-resistant S. aureus; SCCmec: staphylococcal cassette chromosome mec. } \\
\text { †Generated by using Cox proportional hazards regression. } \\
\text { †Categorized into no S. aureus colonization (0), MSSA colonization (1) and MRSA colonization (2), to generate hazard ratios for MSSA colonization (1) } \\
\text { and MRSA colonization (2) in reference to no S. aureus colonization (0). In the analysis by SCCmec types, exposure was categorized into: no S. aureus } \\
\text { colonization (0), MSSA colonization (1), MRSA SCCmec II (2), and MRSA SCCmec IV (3) and hazard ratios for MRSA SCCmec II (2), and MRSA } \\
\text { SCC mec IV were reported. In colonized participants, the analysis was repeated with MSSA colonization being the reference category. } \\
\text { §Adjusted for age, sex, race/ethnicity, and Poverty Income Ratio. } \\
\text { TAdjusted for age, sex, race/ethnicity, poverty Income ratio, smoking, body mass index, and co-morbidities (asthma, diabetes, hypertension, } \\
\text { cardiovascular diseases, stroke, liver disease, and cancer). }\end{array}$} \\
\hline
\end{tabular}

Delayed treatment initiation, virulence factors such as PVL, or both have been hypothesized to be responsible for excess mortality in MRSA infections and bacteremia. Subsequent research found that PVL was associated with decreased time from colonization to bacteremia but not to mortality (9). Again in confirmation of our results, Han et al. studied the effect of SCCmec type on the 30-day inhospital mortality in 184 patients with MRSA and found SCCmec II significant in univariate analysis, but not after co-variates adjustment (10). That study also concluded that SCCmec II was a marker for disease severity instead of a predictor of mortality.

Our study had several limitations. The carriage of $S$. aureus could be transient in some cases and not always reflect the carriage pattern over time. Although the nares are the most common site for $S$. aureus carriage, extranasal sites can also harbor the organism (11). S. aureus carriage was tested in 2001-2004 and might not reflect the current epidemiology and MRSA strain types. Data on co-morbidities, such as cardiovascular or liver diseases and cancer, were not available for participants 18-19 years of age and were coded as missing in the analyses. We did not compare death for the genes encoding enterotoxins because of their limited numbers. However, these NHANES data are the only national data available, and the major strengths of our study include the sample size representative of the US population with generalizable findings and higher power for statistical inferences.

Our findings are of public health and clinical importance because a lack of independent association between colonization by $S$. aureus and mortality implies that decolonization might not prevent death. Some studies indicate that decolonization of patients at high risk for infections could reduce the number of subsequent $S$. aureus infections, but evidence remains limited that it prevents death (5).

Dr. Mendy is a physician completing a PhD in epidemiology at the University of Iowa. His research focuses on infectious causes of chronic diseases.

\section{References}

1. Yaw LK, Robinson JO, Ho KM. A comparison of long-term outcomes after meticillin-resistant and meticillin-sensitive Staphylococcus aureus bacteraemia: an observational cohort study. Lancet Infect Dis. 2014;14:967-75. http://dx.doi.org/10.1016/S14733099(14)70876-X

2. Rose HR, Holzman RS, Altman DR, Smyth DS, Wasserman GA, Kafer JM, et al. Cytotoxic virulence predicts mortality in nosocomial pneumonia due to methicillin-resistant Staphylococcus aureus. J Infect Dis. 2015;211:1862-74. http://dx.doi.org/10.1093/infdis/ jiu554

3. Wertheim HF, Vos MC, Ott A, van Belkum A, Voss A, Kluytmans JA, et al. Risk and outcome of nosocomial Staphylococcus aureus bacteraemia in nasal carriers versus non-carriers. Lancet. 2004;364:703-5. http://dx.doi.org/10.1016/S0140-6736(04)16897-9

4. Nguyen MH, Kauffman CA, Goodman RP, Squier C, Arbeit RD, Singh N, et al. Nasal carriage of and infection with Staphylococcus aureus in HIV-infected patients. Ann Intern Med. 1999;130:221-5. http://dx.doi.org/10.7326/0003-4819-130-3-199902020-00026

5. Schweizer ML, Bossen A, McDanel JS, Dennis LK. Staphylococcus aureus colonization before infection is not associated with mortality among $S$. aureus-infected patients: a meta-analysis. Infect Control Hosp Epidemiol. 2012;33:796-802. http://dx.doi.org/10.1086/666628

6. Niclaes L, Buntinx F, Banuro F, Lesaffre E, Heyrman J. Consequences of MRSA carriage in nursing home residents. Epidemiol Infect. 1999;122:235-9. http://dx.doi.org/10.1017/ S0950268898001770

7. Manzur A, De Gopegui ER, Dominguez M, Mariscal D, Gavalda L, Perez JL, et al.; Spanish Network for Research in Infectious Diseases. Clinical significance of methicillin-resistant Staphylococcus aureus colonization in residents in community long-term-care facilities in Spain. Epidemiol Infect. 2012;140:400 6. http://dx.doi.org/10.1017/S0950268811000641

8. Chan TC, Cheng VC, Hung IF, Chan FH, Ng WC, Yuen KY. The association between methicillin resistant staphylococcus aureus 
colonization and mortality in Chinese nursing home older adults: a 2-year prospective cohort. J Am Med Dir Assoc. 2015;16:796-7. http://dx.doi.org/10.1016/j.jamda.2015.05.020

9. Blaine KP, Tuohy MJ, Wilson D, Procop GW, Tisch DJ, Shrestha NK, et al. Progression to bacteremia in critical care patients colonized with methicillin-resistant Staphylococcus aureus expressing Panton-Valentine leukocidin. Diagn Microbiol Infect Dis. 2010;68:28-33. http://dx.doi.org/10.1016/j. diagmicrobio.2010.04.013

10. Han JH, Edelstein PH, Bilker WB, Lautenbach E. The effect of staphylococcal cassette chromosome mec (SCCmec) type on clinical outcomes in methicillin-resistant Staphylococcus aureus bacteremia. J Infect. 2013;66:41-7. http://dx.doi.org/10.1016/j. jinf.2012.09.001

11. Wertheim HF, Verveer J, Boelens HA, van Belkum A, Verbrugh HA, Vos MC. Effect of mupirocin treatment on nasal, pharyngeal, and perineal carriage of Staphylococcus aureus in healthy adults. Antimicrob Agents Chemother. 2005;49:1465-7. http://dx.doi.org/10.1128/AAC.49.4.1465-1467.2005

Address for correspondence: Angelico Mendy, Department of Epidemiology, The University of Iowa, College of Public Health, S161 CPHB, 105 River St, Iowa City, IA 52242, USA; email: angelico-mendy@uiowa.edu

\section{April 2015: Emerging Viruses}

- Reappearance of Chikungunya, Formerly Called Dengue, in the Americas

- Hantavirus Pulmonary Syndrome, Southern Chile, 1995-2012

- Animal-Associated Exposure to Rabies Virus among Travelers, 1997-2012

- Evolution of Ebola Virus Disease from Exotic Infection to Global Health Priority, Liberia, Mid-2014

- Population Structure and Antimicrobial Resistance of Invasive Serotype IV Group B Streptococcus, Toronto, Ontario, Canada

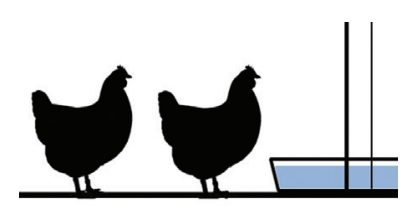

- Sequence Variability and Geographic Distribution of Lassa Virus, Sierra Leone

- Norovirus Genotype Profiles Associated with Foodborne Transmission, 1999-2012

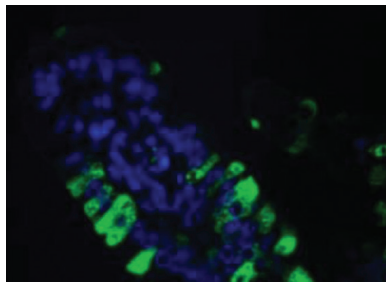

- Deaths Associated with Respiratory Syncytial and Influenza Viruses among Persons $>5$ Years of Age in HIVPrevalent Area, South Africa, 1998-2009

- Influenza A(H7N9) Virus Transmission between Finches and Poultry

- Highly Pathogenic Avian Influenza $\mathrm{A}(\mathrm{H} 5 \mathrm{~N} 1)$ Virus Infection among Workers at Live Bird Markets, Bangladesh, 2009-2010

- Increased Risk for Group B Streptococcus Sepsis in Young Infants Exposed to HIV, Soweto, South Africa, 2004-2008

- La Crosse Virus in Aedes japonicus japonicus Mosquitoes in the Appalachian Region, United States
- Pathogenicity of 2 Porcine

Deltacoronavirus Strains in Gnotobiotic Pigs

- Multidrug-Resistant Salmonella enterica Serotype Typhi, Gulf of Guinea Region, Africa

- Reassortant Avian Influenza A(H9N2) Viruses in Chickens in Retail Poultry Shops, Pakistan, 2009-2010

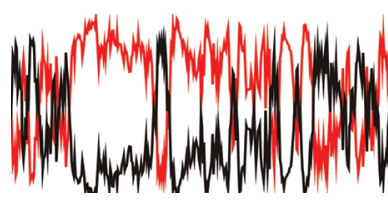

- Candidate New Rotavirus Species in Sheltered Dogs, Hungary

- Severity of Influenza $\mathrm{A}(\mathrm{H} 1 \mathrm{~N} 1)$ Illness and Emergence of D225G Variant, 2013-14 Influenza Season, Florida, USA

- Close Relationship of Ruminant Pestiviruses and Classical Swine Fever Virus

- Peste des Petits Ruminants Virus in Heilongjiang Province, China, 2014
- West Nile Virus Infection Incidence Based on Donated Blood Samples and Neuroinvasive Disease Reports, Northern Texas, 2012

- Influenza A(H10N7) Virus in Dead Harbor Seals, Denmark

- Spotted Fever and Scrub Typhus Bacteria in Patients with Febrile Illness, Kenya

- Virus Antibodies, Israel, 2009-2010

- Outbreak of Severe Zoonotic Vaccinia Virus Infection, Southeastern Brazil

- Lack of Middle East Respiratory Syndrome Coronavirus Transmission from Infected Camels

- Safety of Recombinant VSV-Ebola Virus Vaccine Vector in Pigs

- Enterovirus 71 Subgenotype B5, France, 2013

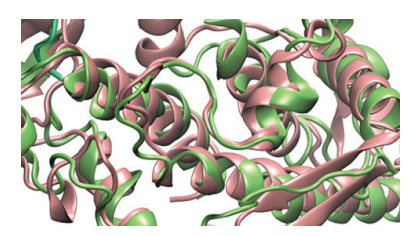

